\title{
Motor Fault Detection and Diagnosis Based on Negative Selection Algorithm
}

\author{
Lihua Zhou, a , Zhongjian Dai ${ }^{2, b}$, Yaping Dai ${ }^{3, c}$ and Linhui Zhao ${ }^{* 4, d}$ \\ ${ }^{1,2,3}$ School of Automation, Beijing Institute of Technology, Beijing, China \\ ${ }^{*} 4$ School of Mechatronics, Beijing Union University, Beijing, China \\ a947280080@qq.com, bpadzj@sina.com, cdaiyaping@bit.edu.cn*djtlinhui@buu.edu.cn
}

Keywords: artificial immune system; negative selection algorithm; fault detection; fault diagnosis

\begin{abstract}
This paper presents a motor fault diagnosis method based on negative selection algorithm. It has the structure of two-level detectors, the first level detector detecting the presence of faults and the second level detector detecting the type of faults. Therefore the first level detectors are trained by using motor normal signals, and the second level detectors are trained by using several types of fault signals. During the process of detecting, only the test results of first level detectors are abnormal, the second level detectors are activated and implement fault detection to identify fault type. In this paper, normal vibration signals of motor bearing and three types of fault signals from American Case Western Reserve University bearing fault database are used to verify the fault diagnosis method. The experimental results show that the method can effectively detect early failure and can correctly identify the fault type.
\end{abstract}

\section{Introduction}

Motor is a kind of indispensable production equipment in petrochemical, metallurgy, electric power and aerospace industries, and it is widely used in a variety of environments. Harsh environments, misuse and dynamic loads and other factors are the main reason for the wear of the motor [1]. Fault diagnosis is an important modern automated production means to improve system reliability and security [2]. With the development of the past decades, fruitful results are achieved in this field.

Artificial immune system is a biological immune system simulation with powerful information processing capability, it is a computing paradigm inspired by biological theory, draws on some features, principles and models of the immune system for judgment and decision-making of complex problems [3]. Over the past decade, researchers have proposed artificial immune network model, artificial immune system application framework model, general immune algorithm, negative selection algorithm (NSA), clonal selection algorithm and immune learning algorithm, which are successfully applied in many research fields [4]. Compared with the conventional method of rotating machinery fault diagnosis, artificial immune system has advantages of providing noise tolerance, unsupervised learning, self-organization, articulate knowledge, forgetting seldom-used knowledge and content accessible memory, etc. It has the advantage of distributed parallel processing and robustness at the same time [5].

This paper proposes a motor fault diagnosis method based on NSA with the structure of two-level detectors. The normal signals of motor are used to generate first-level detectors for detecting the presence of faults. There is no necessary for any prior knowledge of faults at this stage. The fault signals of motor are used to generate second-level detectors. When a fault is detected, second-level detectors can identify the fault type. The proposed fault diagnosis method has a hierarchical structure, and motor bearing data is used to validate the effectiveness of the method.

The paper is organized as follows: Section 2 describes the basic principle of NSA, section 3 describes the proposed motor fault diagnosis method based on NSA, section 4 shows simulation results, section 5 is the conclusion of this paper. 


\section{Negative Selection Algorithm}

NSA inspired by negative selection mechanism of the immune system is initially proposed by Forrest in 1994 [6]. After that, it is introduced into the fault detection field by Dasgupta, in the application of milling pin tool fracture detection. Basic structure of NSA is shown in figure 1. NSA is divided into offline training phase and online testing phase. In offline training phase, representative normal samples are firstly collected, namely self-sample data set. Then, candidate detectors are generated randomly. Compared with self-sample, only those detectors which don't match all self-samples are reserved, forming mature detector set. Therefore, mature detector set has the ability to detect the nonself-samples. Next, in the online testing phase, test data are compared with all detectors in the mature detector set. If there is any match, then abnormal is detected, otherwise the test data is identified as normal.

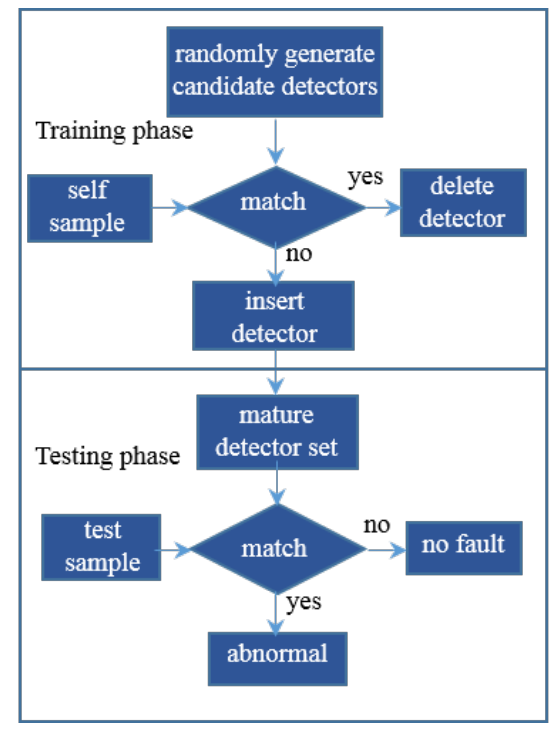

Figure 1. Basic Structure of NSA

In the NSA, the sample space is represented as ${ }^{[0,1]^{n}}, n$ is the sample space dimension, including self-samples and nonself-samples. Random sampling in the ${ }^{n}$ dimension sample space is considered to be the center of candidate detector. Self-sample and candidate detector are expressed as $\left[x_{1}, x_{2}, \cdots, x_{n}\right]$ and $\left[d_{1}, d_{2}, \cdots, d_{n}\right]$. The affinity between self-sample and candidate detector is represented by the Euclidean distance, as shown in equation (1). Match error $H$ is the difference between affinity $d$ and predetermined threshold $\lambda$, as shown in equation (2). When $H<0$, the candidate detector is matched with self-samples. When $H>0$, the candidate detector is not matched with self-samples, then is inserted into the mature detector set. There are two criteria to stop generating detectors, reaching a predetermined number or reaching a predetermined coverage. This paper takes the criteria of reaching a predetermined number. The predetermined detector number is determined according to the best diagnostic results in a series of experiments.

$$
d=\sqrt{\sum_{i=1}^{n}\left(x_{i}-d_{i}\right)^{2}} .
$$

$H=d-\lambda$.

In the NSA, high coverage of the detectors will significantly increase the number of detectors. However, low coverage can't fully cover the non-self region, which will lead the decrease in fault detection rate. In the NSA proposed by Forrest, number of detectors will increase exponentially with normal space. The coverage in fault space is difficult to balance with the detector overlap, limiting the application of such negative algorithm. There have been many NSA variants in recent years, one of which is the $\mathrm{V}$-detector algorithm. It can reduce the number of detectors under the premise of 
coverage, and is widely used in fault detection. Therefore the V-detector algorithm is adopted in this paper. Figure 2 is the detector generating process in the $\mathrm{V}$-detector algorithm. Figure 3 is the detection process using $\mathrm{V}$-detector algorithm.

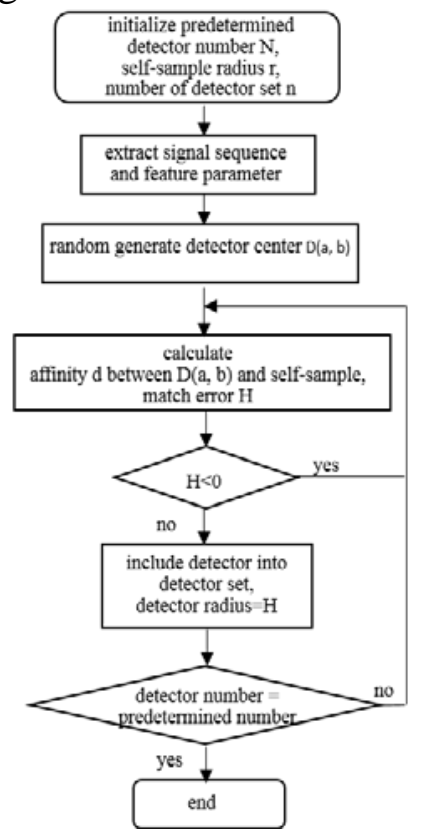

Figure 2. Detector Generating Process in V-detector Algorithm

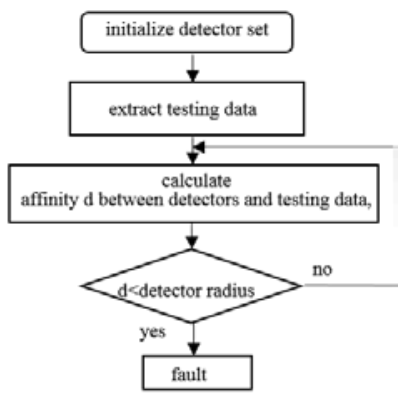

Figure 3. Detection Process using V-detector Algorithm

\section{Motor Fault Diagnosis Method based on NSA}

Fault diagnosis is considered to be more complex than fault detection, since it is not only able to detect any presence of early failures, but also able to correctly identify the fault type [7]. The traditional NSA can only identify normal and fault status of the motor signals, but can't determine the fault type.

In this paper, motor vibration signals are used for fault detection and diagnosis. First, signals are processed so that to be mapped into $n$ dimensional space ${ }^{[0,1]^{n}}$. The motor signal sample space includes self-samples and nonself-samples, in which nonself-samples include all kinds of fault samples. Self-sample is expressed as $S$, and nonself-sample is expressed as $N S$.All kinds of fault samples are expressed as $F_{1}, F_{2}, \ldots, F_{n}$.The relationship between them are shown in Figure 4.

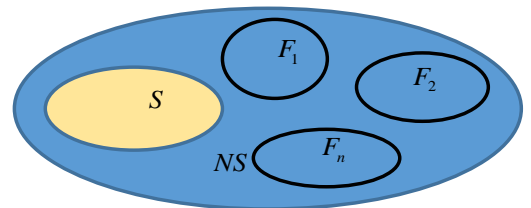

Figure 4. Relationship between Self-samples, Nonself-samples and Fault samples

In the proposed algorithm, normal vibration signals are used to train the first-level detector set, which is able to correctly identify self-samples and nonself-samples. Fault vibration signals are used to train the second-level detector set, which can identify fault and non-fault samples. Assume those 
detectors trained by normal vibration signals are represented as $D_{s}$, and those detectors trained by fault vibration signals are represented as $D_{f 1}, D_{f 2}, \ldots, D_{f n}$. When a sample is matched with any detector, the detection result is expressed as 1 , otherwise the test result is expressed as 0 . When the results of the first level detector set are all 1, then the second level detector is triggered to determine the type of fault. Fault diagnosis decision of two-level detection is shown in Table 1. For example, when the test data is second fault type, the detection result of first-level detector is 1 , so the second-level detectors is triggered, according to the matching rules, the results of second-level detectors are $1,0,1, \ldots, 1$, therefore the diagnosis result is second fault type.

Table 1. Two-Level Detector Fault Diagnosis Decision

\begin{tabular}{|c|c|c|c|c|c|c|}
\hline Detector & \multicolumn{6}{|c|}{ Detection Result } \\
\hline$D_{s}$ & 0 & 1 & 1 & & 1 & 1 \\
\hline$D_{f 1}$ & & 0 & 1 & $\cdots$ & 1 & 1 \\
\hline$D_{f 2}$ & & 1 & 0 & $\ldots$ & 1 & 1 \\
\hline$\vdots$ & & $\vdots$ & $\vdots$ & $\ddots$ & $\vdots$ & $\vdots$ \\
\hline$D_{f n}$ & & 1 & 1 & $\ldots$ & 0 & 1 \\
\hline Diagnosis Result & No Fault & Fault1 & Fault 2 & $\ldots$ & Fault $\mathrm{n}$ & Unknown fault \\
\hline
\end{tabular}

\section{Simulation Results}

In this paper, American Case Western Reserve University bearing fault database is used for testing the proposed diagnosis method. Experimental data include normal vibration signals, inner ring fault signals, ball fault signals and outer ring fault signals. The fault size is 0.007 inches, and motor speed is $1797 \mathrm{rmp} / \mathrm{s}$. Their time domain vibration signals are shown in Figure 5.

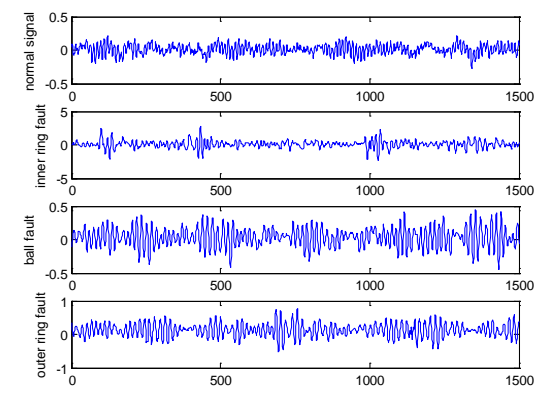

Figure 5. Vibration Waveform of Normal Signal and Fault Signal

The calculation amount is reduced by limiting the data window length. In this paper, data window length is selected as 512, namely 512 discrete points form a signal sequence. According to the Western Reserve bearing database, 250 signal sequences are extracted, and then the characteristic parameters are extracted from the signal sequence. The probability density function can be obtained by vibration amplitude, deriving dimension index parameters. The dimension index is sensitive to the fault, load, speed and other conditions. Dimensionless indicators are composed of a ratio of two components with the same dimension, and it's not sensitive to interference, but it is difficult to search the relationship between the characteristics of fault [8]. In this paper, the signal sequence is processed by using dimensional index-mean and dimensionless index-kurtosis. The mean and kurtosis index are shown as equation (3) and (4). Two-dimension feature parameters are mapping into feature space by using the linear normalization equation, as shown in figure 6.

$$
\bar{X}=\frac{1}{n} \sum_{i=1}^{n} m_{i} .
$$




$$
Y=\frac{\frac{1}{n} \sum_{i=1}^{n} m_{i}{ }^{4}}{\left[\frac{1}{n} \sum_{i=1}^{n} m_{i}{ }^{2}\right]^{2}} .
$$

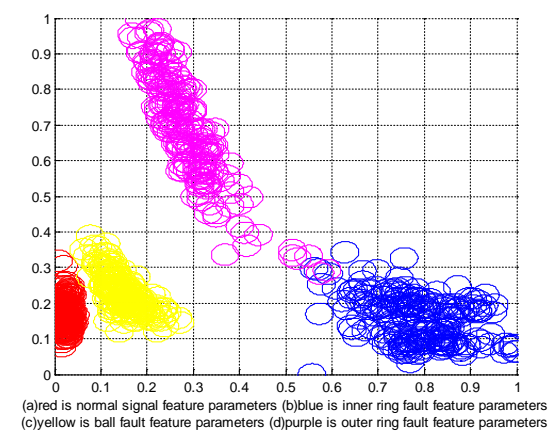

Figure 6. Feature Parameters

The first-level detector set and the second- level detector set are trained using the normalized normal signal characteristic parameters and fault signal characteristic parameters. After the first-level detector set identifies a failure, the second-level detector set is activated to diagnose fault type. All normal and fault detector sets are shown in Figure 7. In the experiment, self-sample radius is selected as 0.03 , the number of detector set is 1000 . Fault detection and fault diagnosis rate and average detection time are shown in Table 2.

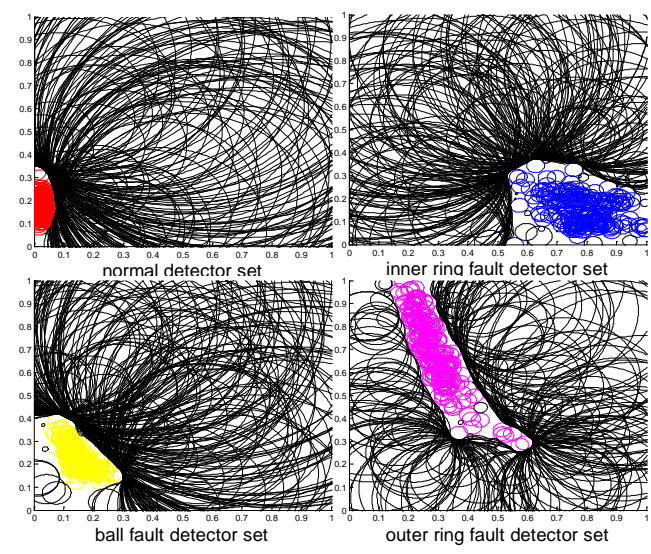

Figure 7. Normal and Fault Detector Set

Table 2. Fault Detection and Fault Diagnosis Result

\begin{tabular}{|c|c|c|c|c|}
\hline & Normal Data & Inner Ring Fault & Ball Fault & Outer Ring Fault \\
\hline Accuracy Rate & $97.43 \%$ & $94.39 \%$ & $95.72 \%$ & $95.53 \%$ \\
\hline Diagnosis Time & $9.87 \mathrm{~ms}$ & $8.76 \mathrm{~ms}$ & $9.09 \mathrm{~ms}$ & $8.26 \mathrm{~ms}$ \\
\hline
\end{tabular}

In order to verify the performance of fault diagnosis system based on NSA, the diagnose fault algorism based on BP neural network is experimented with the same data. The input layer of BP neural network has 2 nodes, and the output layer has 4 nodes. Four experiments are taken with the different number of hidden layer node, selecting respectively 5, 10, 15 and 20. Training data include normal motor bearings data and three kinds of fault data, and each of them has 200 samples. Testing data is composed of 50 samples of normal motor bearings data and 150 samples of three kinds of fault data. Diagnosis results are shown in table 3.

Table 3. BP Neural Network’s Fault Diagnosis Accuracy Rate

\begin{tabular}{|c|c|c|c|c|}
\hline Hidden Layer Node & 5 & 10 & 15 & 20 \\
\hline Normal Data & $69.71 \%$ & $78.43 \%$ & $64.89 \%$ & $65.89 \%$ \\
\hline Inner Ring Fault & $99.81 \%$ & $99.80 \%$ & $99.84 \%$ & $99.28 \%$ \\
\hline Ball Fault & $35.40 \%$ & $28.76 \%$ & $44.63 \%$ & $39.21 \%$ \\
\hline Outer Ring Fault & $94.30 \%$ & $94.38 \%$ & $94.08 \%$ & $93.36 \%$ \\
\hline
\end{tabular}


Experiment results show that the approach proposed in this paper can meet the requirements for real-time detection and diagnosis, and can correctly diagnose the fault type. Comparing table III and table IV, we can find that comprehensive fault diagnosis accuracy rate of the proposed NSA algorithm is higher than the BP neural network's. It can be seen that NSA's diagnostic rate of normal data, ball fault data and outer ring fault data are higher than BP neural network, but the inner ring fault diagnostic rate in negative selection algorithm is lower than BP neural network, the reason for this result remains to be further analyzed.

\section{Summary}

A motor fault diagnosis method based on NSA is presented in this paper, which has a hierarchical structure, in which fault detection and fault diagnosis are conducted respectively in the first and second stages. The method is independent of motor type, therefore, it can be extended to more types of fault diagnosis. In this paper, normal motor bearing signals and a variety of fault signals are used to the test the method, verifying the correctness and practicability of the diagnosis system. The fault diagnosis method based on BP neural network is compared with the proposed method. The experimental results show that fault detection rate of the proposed method reached $97.43 \%$, and the diagnosis rate of bearing inner ring fault, ball fault and outer ring fault reached $94.39 \%, 95.72 \%$ and 95.53\%, which means the comprehensive diagnosis performance is better than BP neural network. It is proved the fault diagnosis algorithm based on NSA has practical value and popularization.

In the actual production process, there will appear new unpredictable fault type, and the fault characteristic center offset will occur. Since the proposed algorithm is a static approach, so dynamically adjust fault center, adding new fault type are the next focal point of the work. Also, the diagnosis accuracy rate can be further improved by analyze the data pattern adaptive to neural network and make a fusion diagnosis strategy of the negative selection algorithm and neural network.

\section{Acknowledgment}

This work was financially supported by the Importation and Development of High-Caliber Talents Project of Beijing Municipal Institutions (CIT\&TCD201404081).

\section{References}

[1] Wen X, Liao Q, Wei S, et al. Application Research of Immune Neural Network on Motor Fault Diagnosis[J]. Education Technology \& Training \& International Workshop on Geoscience \& Remote Se, 2008, 1:618-621.

[2] Zhou D H, Yan-Yan Hu, et al. Fault Diagnosis Techniques for Dynamic Systems[J]. Acta Automatica Sinica, 2009, 35(6), 748-758.

[3] Abbass H A, Amin R, Sarker C S N. Data mining: a heuristic approach, 2002, Idea[J]. Online Information Review, 2002, 27(5):364-365.

[4] Zhang, Q. H. (2004). Fault diagnosis technology research based on artificial immune system. Guangzhou: South China University of Technology. (in Chinese)

[5] Yue X, Wen D, Ma H, et al. Fault Detection Based on Real-Value Negative Selection Algorithm of Artificial Immune System[C]// Proceedings of the 2010 International Conference on Intelligent Computing and Cognitive Informatics. IEEE Computer Society, 2010:243-246.

[6] Forrest S, Perelson A S, Allen L, et al. Self-Nonself Discrimination in a Computer[C]// In Proceedings of the 1994 IEEE Symposium on Research in Security and Privacy. 1994:202--212.

[7] Yuan G L, Qin S W, Zhang J. Fault Diagnosis of Induction Motor Based on Artificial Immune System[C]// Industrial Control and Electronics Engineering (ICICEE), 2012 International Conference on. IEEE, 2012:166 - 170.

[8] Cen J, Zhang Q H, Xu B G, et al. Fault Diagnosis Model of Rotating Machinery Based on Artificial Immunity and Its Application[C]// Intelligent Systems and Applications, 2009. ISA 2009. International Workshop on. IEEE, 2009:1 - 4. 